\title{
Acondicionamiento de señal de sensor infrarrojo
}

\section{Conditionated infrared sensor}

\author{
MARTINEZ-REYES, Armando $\dagger^{*}$, RANGEL-GOMEZ, Irving, GARCIA-ANDRADE, Roxana y \\ FERNANDEZ-RAMIREZ, Arnoldo
}

Tecnológico Nacional de México/Instituto Tecnológico de Nuevo León

ID 1 ${ }^{\mathrm{er}}$ Autor: Armando, Martinez-Reyes / ORC ID: 0003-2507-405X, CVU CONACYT ID: 1016002

ID $1^{\text {er }}$ Coautor: Irving, Rangel-Gomez / ORC ID: 0003-2567-5525, CVU CONACYT ID: 816580

ID $2^{\text {do }}$ Coautor: Roxana, Garcia-Andrade / ORC ID: 0000-0003-2819-6482, CVU CONACYT ID: 1015497

ID $3^{\text {er }}$ Coautor: Arnoldo, Fernandez-Ramirez, ORC ID: 00001-7906-9307

DOI: $10.35429 / J B E B .2019 .8 .3 .1 .7$

Recibido 22 de Febrero, 2019; Aceptado 30 Junio, 2019

\section{Resumen}

En el presente trabajo se realizó el diseño e implementación de un dispositivo capaz de monitorear el pulso cardiaco por medio de sensores fotoeléctricos, utilizando la técnica de oximetría y a su vez la fotometría. El monitoreo del ritmo cardiaco tiene gran relevancia en el campo de la salud y la tecnología, debido a la gran cantidad de enfermedades cardiovasculares y las millones de muertes anuales a nivel mundial. Actualmente existen diversos contadores del ritmo cardíaco con limitantes que no pueden enviar su información vía internet o GPRS, y no puede ser archivada en una computadora, Este prototipo se realizó, basado en un microcontrolador que recibe una señal de sensor infrarrojo, una vez acondicionada adecuadamente se podrá transmitir a una computadora. La información obtenida podrá ser analizada y almacenada por especialistas médicos, así como también transmitirla por wifia una aplicación de un dispositivo remoto dando un servicio con el aprovechamiento de las tecnologías.

Foto sensores, Pulso cardiaco, Remoto

\begin{abstract}
In the present work, the design and implementation of a device capable of monitor the cardiac pulse by means of photoelectric sensors was carried out, using the oximetry technique and in turn the photometry. Heart rate monitoring has great relevance in the field of health and technology, due to the large number of cardiovascular diseases and millions of deaths worldwide annually. There are currently several heart rate counters with limitations that cannot send your information via internet or GPRS, and cannot be filed on a computer. This prototype was made, based on a microcontroller that receives an infrared sensor signal, once properly conditioned It can be transmitted to a computer. The information obtained can be analyzed and stored by medical specialists, as well as transmitted by Wi-Fi to an application of a remote device providing a service with the use of technologies.
\end{abstract}

Photo sensor, Heart rate, Remote

Citación: MARTINEZ-REYES, Armando, RANGEL-GOMEZ, Irving, GARCIA-ANDRADE, Roxana y FERNANDEZRAMIREZ, Arnoldo. Acondicionamiento de señal de sensor infrarrojo. Revista de Ingeniería Biomédica y Biotecnología. 2019. 3-8: 1-7

\footnotetext{
* Correspondencia del autor (Correo electrónico: armando2k@ hotmail.com)

$\dagger$ Investigador contribuyendo como primer autor
} 


\section{Introducción}

A lo largo de la historia de la Humanidad, se han ido produciendo grandes avances en el ámbito de la ciencia para satisfacer las necesidades de las personas. Más concretamente desde el punto de vista médico, la ingeniería ha sustentado un papel primordial en el avance de muchos aspectos, como pueden ser el desarrollo de técnicas preventivas, aplicaciones o prototipos que puedan hacer frente a distintos problemas que han acontecido a las personas desde siempre. Cabe destacar también el papel que tiene a día de hoy la electrónica, ya que permite el desarrollo de distintos dispositivos que permiten que los especialistas puedan obtener datos de distintos pacientes mediante técnicas no invasivas.

Hace 100 años los científicos intentaron relacionar la temperatura con la longitud de onda. Esto permitió reconocer que la porción del espectro denominado infrarrojo se localiza en un punto donde no se hace presente la luz visible al ojo humano. En 1830 se identificó que los cuerpos emiten radiación termal y de esta forma se experimentó con instrumentos para medir temperatura. Schlessinger, M. (2019).

En la última década, la fabricación en masa de distintos componentes electrónicos ha dado lugar a que, en el ámbito de la biotecnología, se pueda hacer uso de distintos elementos para poder desarrollar pequeños dispositivos que permitan obtener datos biomédicos como es el caso de los pulsioximetros. Incluso, se estima que en los años siguientes siga habiendo un aumento del desarrollo de este tipo de dispositivos lo que influirá positivamente en la recolección de nuevas técnicas de desarrollo para poder contrarrestar cualquier tipo de anomalía en el ámbito de la salud.

Concretamente este trabajo tiene como objetivo la implementación de un prototipo basado en microcontroladores, obteniendo datos biomédicos por medio de un sensor $\mathrm{y}$ visualizándolos por medio de una computadora a su vez poder comparar esta información con las gráficas del Dr. Poincare. Es por esto que, con este trabajo se busca el desarrollo de un servicio que pueda ser utilizado por la mayoría de la población y que funcione como un "sistema de alarma " para que toda aquella persona en situación de riesgo pueda detectar, un problema a tiempo.

\section{Antecedentes}

Este trabajo se inició conociendo dos principios de medición de pulso cardiaco. Siendo estos los siguientes:

\section{Pulsos eléctricos}

Desde épocas remotas el control de la frecuencia cardiaca se realizó por cálculos parciales recurriendo a escalas de magnitud del esfuerzo generalizado o en el mejor de los casos establecidas de manera instintiva por el personal profesional de la salud. El control era cercano al real y por tanto el parámetro principal era la experiencia y práctica del médico. Un gran descubrimiento en el campo de la medicina es el registro de la función eléctrica del corazón, asi como en los tejidos humanos y animales, llegando a la conclusión que un latido siempre iba acompañado de un pequeño pulso eléctrico.

Así a finales del siglo XIX aparece el primer electrocardiograma realizado con un galvanómetro por Auguste Waller figura 1. Quedando definidas las señales para el electrocardiograma P, Q, R, S y T, y es Willem Einthoven el que emplea el galvanómetro para realizar mediciones. Al pasar los años el electrocardiograma se va perfeccionando hasta llegar a la actualidad que se cuenta con un Holter. Medran, R. (2018)

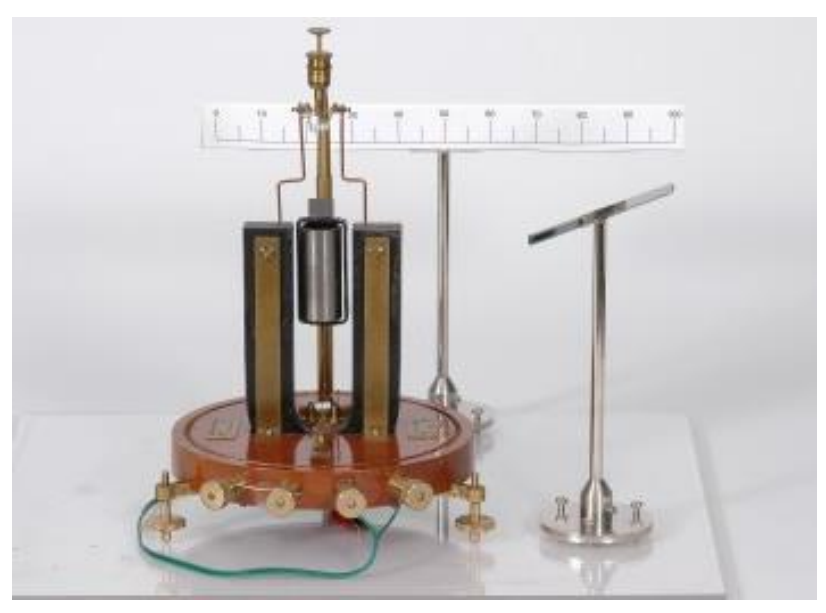

Figura 1 Galvanómetro D'Arsonval

Fuente: http:/biblog.etsit.upm.es

\section{Oximetría}

La oximetría de pulso es un método que permite la estimación de la saturación de oxígeno de la hemoglobina arterial y también vigila la frecuencia cardiaca y la amplitud del pulso. 
Los primeros avances en el concepto de la oximetría fueron realizados en el año 1918 durante la primera Guerra Mundial cuando Krogh en Copenhague intento medir la oxigenación de pilotos. En 1930 Millikan y Wood desarrollaron un oxímetro de pabellón auricular de dos longitudes de onda y en 1949 Wood y Geraci pudieron medir la saturación absoluta de oxígeno a través de determinación fotoeléctrica en lóbulo de la oreja.

En 1974, el ingeniero TakuoAyoagi de la NihonKohden, basado en que las pulsaciones arteriales cambian el color de la sangre y pueden ser leídas usando el radio de la absorción de luz roja e infrarroja, desarrolló el primer oxímetro de pulso.

A partir de 1975 el profesor de electrónica de la universidad de Oulu, Finlandia, Seppo Saynajakangas diseñó un sistema telemétrico de Medidor de Ritmo Cardiaco sin hilo, consistente en un receptor de pulso de muñeca, una banda electromagnética de tórax receptora de señales cardiacas y una banda elástica ajustable Saynajakangas investigo durante años el uso de aparatos como el Holter, ECG, unidades foto reflectantes tipo clip para lóbulo de oreja o yemas de dedo etc., y encontró que ningunos de estos aparatos era utilizable para los propósitos deportivos de control del entrenamiento, ya que se requería de un sistema que permitiera obtener, mostrar y calcular los ritmos cardiacos durante sesiones de entrenamiento sin detener el ritmo de práctica.

Para 1977 El profesor Saynajakangas fundo la Polar Electro OY. A su vez en el mismo año 1977 Minolta comercializa el "Oximet" añadiendo dos sensores de fibras ópticas. Posteriormente se realizan ensayos clínicos en la Universidad de Stanford y en 1981 "Biox y Nellcor" añaden los sensores de luz y la señal pulsátil que actualmente se usan en la práctica clínica.

En 1982 obtuvo las primeras patentes de un aparato llamado MRC (medidor Ritmo Cardiaco ECG de corta distancia serie PE 200S). Mejía Salas, Héctor, \& Mejía Suárez, Mayra. (2012)

\section{Desarrollo}

Para la determinación de la saturación de hemoglobina arterial con oxígeno el oxímetro de pulso usa la espectrofotometría basada en que la hemoglobina oxigenada y la hemoglobina reducida absorben y transmiten determinadas longitudes de onda del espectro luminoso para la luz roja (640-660nm) y la luz infrarroja (910$940 \mathrm{~nm}$ ), como se muestra en la figura. 2. La hemoglobina oxigenada absorbe más la luz infrarroja y permite el paso de la luz roja; por el contrario, la hemoglobina reducida absorbe más la luz roja y permite el paso de la luz infrarroja. Aunque la intensidad de la línea de absorción en el infrarrojo cercano a la región espectral es más baja en 2-4 órdenes de magnitud que la del infrarrojo medio la región de los diodos láseres de telecomunicaciones pueden ser atractivos, ya que se pueden operar fácilmente a un bajo costo. Yin, X., Wu, H., Dong, L., Ma, W., Zhang, L., Yin, W., \& Tittel, F. K. (2018).

El radio de la absorción de la luz roja e infrarroja mide el grado de oxigenación de la hemoglobina. La radiación infrarroja tiene la característica de pasar o atravesar un dedo que contiene circulación sanguínea y la absorción cuantitativa de energía podrá ser analizada, por medido por un detector apropiado (detector infrarrojo), en el fotómetro no dispersivo. De Hoyos-Vázquez, F. F., Carreño-de León, M. C., Serrano-Núñez, E. O., Flores-Álamo, N., \& Ríos, M. S. (2019)

Los oxímetros de pulso tienen dos sensores o sondas con diodos emisores de luz uno para luz infrarroja y otro para la luz roja además, de un fotodiodo detector. Para medir el oxígeno los diodos emisores de luz y el fotodiodo detector deben ponerse en puntos opuestos dejando en medio el tejido translucido (pulpejo del dedo, pabellón auricular, etc.).

El mecanismo que permite la lectura de la oxigenación es que en cada pulsación de la sangre arterial se transmiten valores lumínicos, detectando al mismo tiempo la frecuencia cardiaca. Asumiendo que solo la sangre arterial pulsa a esto se denomina componente arterial pulsátil. La cantidad de luz absorbida cambia de acuerdo a la cantidad de sangre en las células y la presencia de hemoglobina oxigenada/hemoglobina reducida. 
Por otro lado existe un componente estático que está formado por los tejidos, huesos, piel y la sangre venosa.

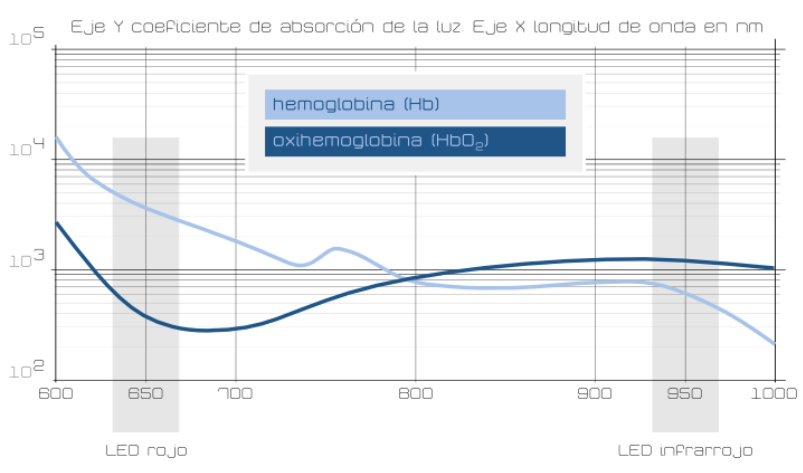

Figura 2 Frecuencia de led rojo e infrarrojo Fuente: Ventura, V. (2015)

Este diseño está basado en la plataforma Arduino el sistema operativo y los programas que corren sobre el no requieren pagos de licencias. Se contempla la utilidad de este prototipo para personas que no pueden trasladarse a un hospital, por su lejana ubicación, o por no disponer de un médico cercano, También se considera ampliar el número de mediciones ya que el microcontrolador tiene capacidad para monitorear más variables según sean los requerimientos médicos.
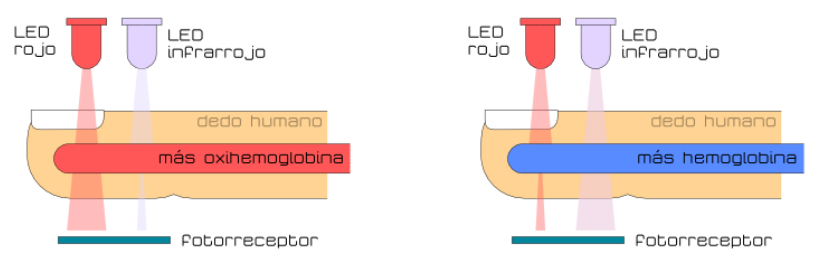

Figura 3 Incidencia de la luz roja e infrarroja Fuente: Ventura, V. (2015)

El microcontrolador requiere en la entrada, un acondicionamiento de señal como preparación para que sea procesado por el microcontrolador, a su vez la entrada del acondicionamiento de señal requiere un sensor que proporcione los datos del latido del corazón mediante la colocación de un sensor de luz en un dedo. Este sensor tendrá un emisor de luz infrarroja a un lado del dedo y al otro lado del dedo tendremos el receptor de esta luz. Como se muestra en la figuras 3 y 4 Según varíe la opacidad de nuestro dedo, la cual depende del oxígeno en la sangre el receptor recibirá más intensidad o menos intensidad de luz que a su vez depende de la frecuencia con la que lata el corazón.

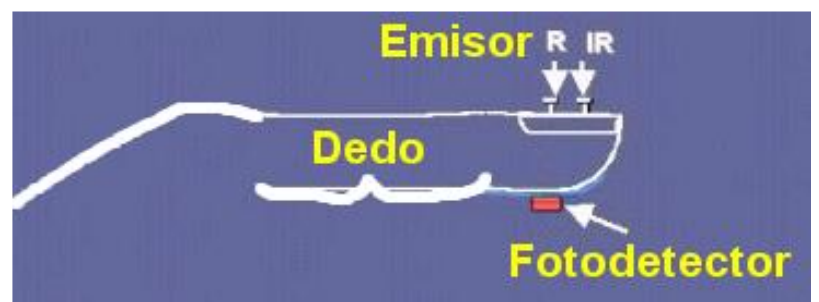

Figura 4 Colocación del sensor en el dedo

Este sensor nos proporcionará una señal eléctrica que mediante un sistema de amplificación y filtros alimentaran a un altavoz y un diodo led los cuales sonarán y se encenderán, una vez por latido, respectivamente.

El acondicionamiento de señal se dividirá en 3 etapas:

La primera es la red compuesta por el diodo led de infrarrojos junto con el fotodiodo, el divisor de tensión de resistencias Rw y Rx que componen el sistema sensor. Este sistema dará un voltaje $\mathrm{Vi}$ en función del voltaje que esté presente resistencia Ri. Esta tensión Vi será proporcional a la intensidad que circula por Ri y al valor de ésta.

La segunda etapa estaría compuesta por el amplificador no inversor que amplifica la señal Ventrada por un cierto valor, dando un voltaje V1 a su salida.

La tercera etapa es un filtro pasa banda que solamente trabajara con frecuencias bajas propias del pulso cardiaco El sistema sensor que comprende el led y el infrarrojo se alimentaron con $5 \mathrm{~V}$, de acuerdo a especificaciones de ambos componente, para poder obtener este valor se realizó un divisor de voltaje, de acuerdo a la hoja de especificaciones del led utilizado LD274, la corriente máxima del diodo es $\mathrm{If}=100 \mathrm{~mA}$, se trabajó con una corriente menor para asegurar que no se llegue al rango de ruptura del diodo, considerando el $\mathrm{V}$ led de $1,1 \mathrm{~V}$ y establecemos una corriente de $25 \mathrm{~mA}$, obtenemos un valor de $\mathrm{R}$ limitadora.

Se observa el circuito en figura 5. La hoja de especificaciones del diodo LD274 tiene una característica, de $50 \mathrm{~mW} / \mathrm{sr}$ para $100 \mathrm{~mA}$. Es decir, cuando por el diodo pasan $100 \mathrm{~mA}$ el fabricante asegura como mínimo de unas potencia lumínica de $50 \mathrm{~mW}$ en el ángulo predeterminado abarcado por el diodo. 
Esta potencia esta referenciada para el valor de $100 \mathrm{~mA}$, se tendrá que dividir entre cuatro ya que la intensidad que se tomo es de $25 \mathrm{~mA}$ y la potencia emitida es directamente proporcional a la intensidad que circula por el led. Por consiguiente le, mínimo $(25 \mathrm{~mA})=$ $12.5 \mathrm{~mW} / \mathrm{sr}$. Es importante considerar el umbral del fotodiodo (BPW34), al ser infrarrojo el rango de longitud de onda en el cual trabaja óptimamente es de 900 a 950nm, es importante considerar esto.

Con esta información podemos calcular el ángulo predeterminado del fotodiodo. El ángulo se define como el área del fotodiodo dividido entre el cuadrado de la distancia que le separa del emisor sabiendo que el área del BPW34 es de $7.5 \mathrm{~mm}^{2}$ y que está separado del diodo led $3 \mathrm{cmluego}$ tendremos el siguiente valor del ángulo rígido, con estos valores se obtiene una fotocorriente de $35 \mu \mathrm{A}$, considerando una frecuencia de 1 hertz ya que es aproximadamente las pulsaciones de persona normal en reposo 60ppm. Mejía, H. \& Mejía, M.(2012)
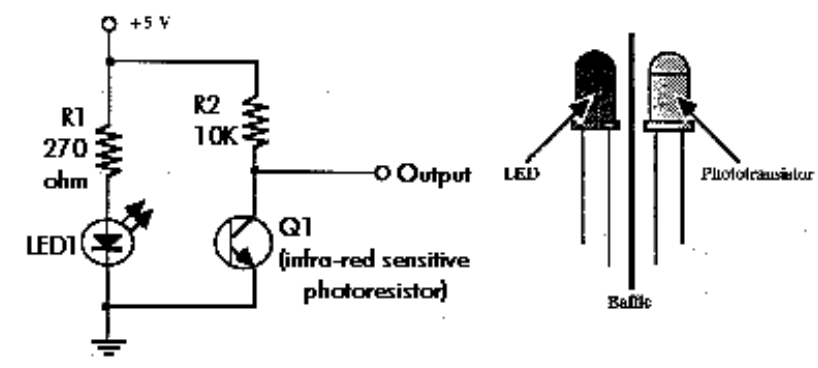

Figura 5 Circuito básico del sensor

En la figura 6 se aprecia la implementación del sensor en una pinza para facilitar el monitoreo.

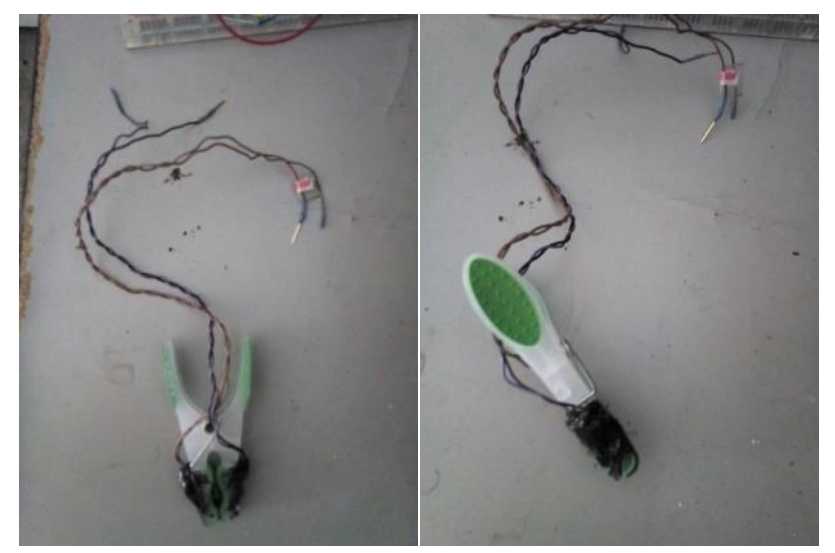

Figura 6 Implementación de sensor de pinza

La siguiente etapa es el amplificador no inversor, en esta sección se construyó un amplificador no inversor ver figura 7 .
En el cual tendremos en la entrada Vi proveniente de la Ri y a la salida, la tensión V1 que estará amplificada. Como tercera etapa del circuito es el filtro pasa banda que limite las frecuencias entre $0.482 \mathrm{~Hz}$ y $4.82 \mathrm{~Hz}$. Boylestad, R \& Nashelsky, L. (2003).

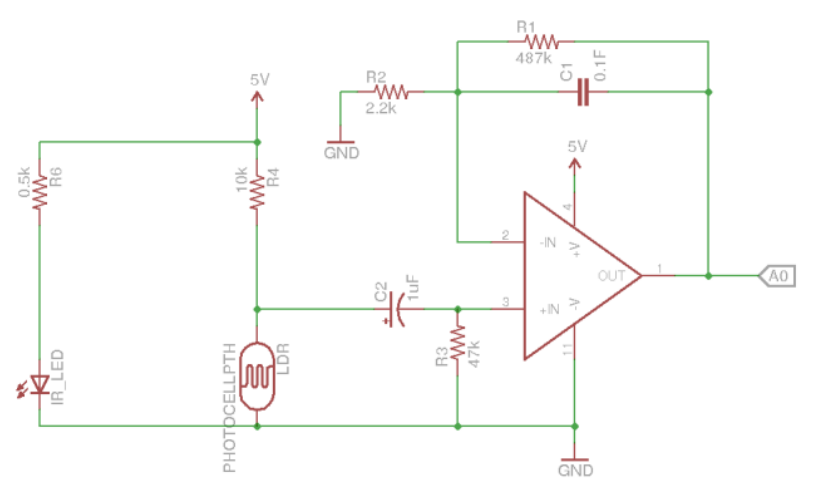

Figura 7 Circuito de prueba

Para la obtención de los pulsos cardiacos en forma gráfica en una computadora se requiere utilizar dos programas que corren simultáneamente, el primero se ejecuta dentro del microcontrolador Arduino el cual recibe la señal del sensor y la envía a través del puerto USB a la computadora. Corona, L., Abarca, G. , Mares, J. (2014). En la computadora se ejecuta un programa basado en el software denominado processing el cual permite recibir la señal del microcontrolador, calcular los latidos por minuto y graficarlos en la pantalla. En la figura 8 y figura 9 se muestra un diagrama de los programas mencionados.

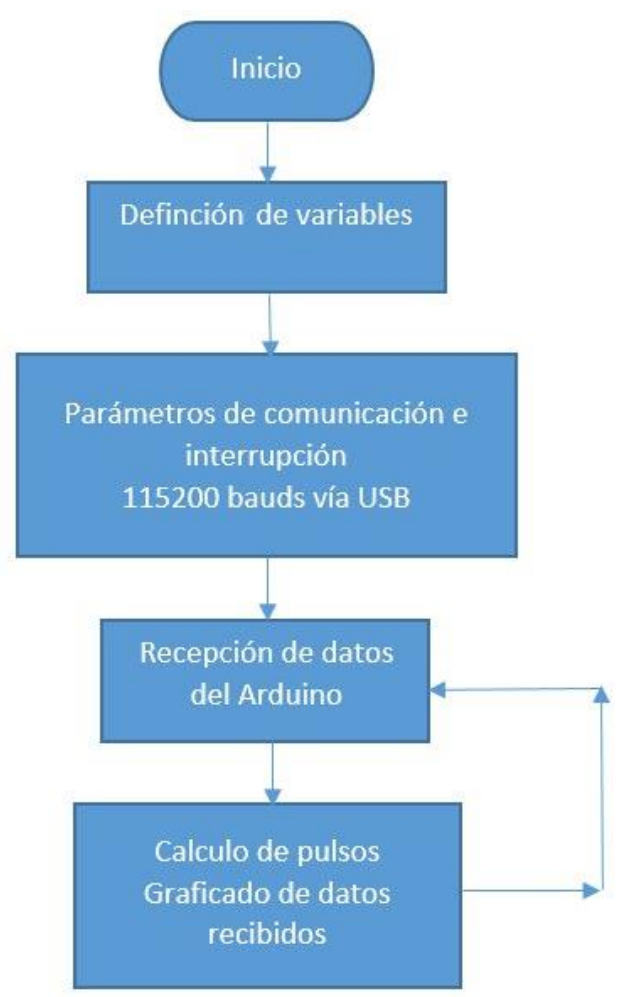

Figura 8 Diagrama de flujo del programa del microcontrolador

MARTINEZ-REYES, Armando, RANGEL-GOMEZ, Irving, GARCIA-ANDRADE, Roxana y FERNANDEZ-RAMIREZ, Arnoldo. Acondicionamiento de señal de sensor infrarrojo. Revista de Ingeniería Biomédica y Biotecnología. 2019 


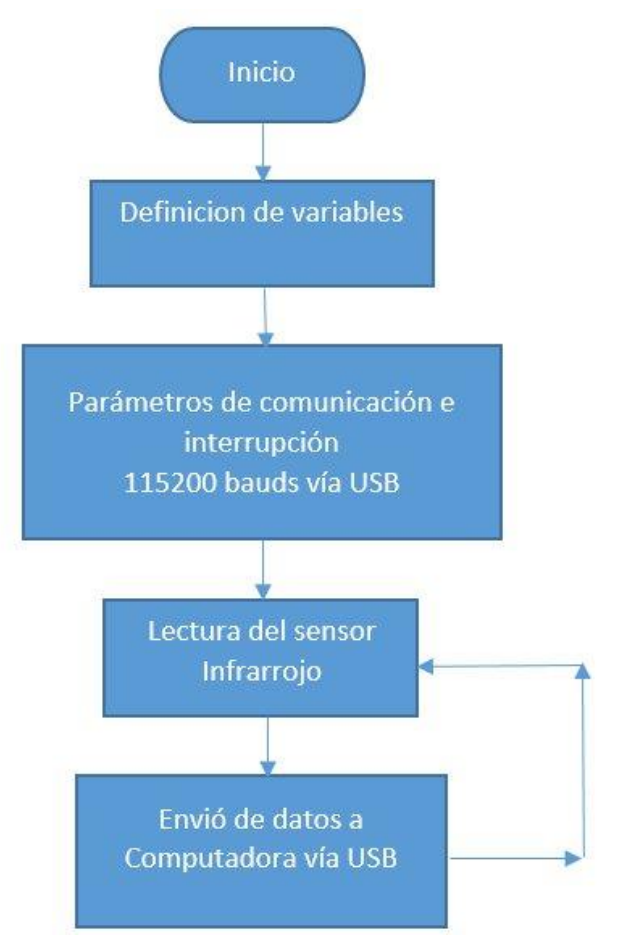

Figura 9 Diagrama de flujo del programa que se ejecuta en la computadora

\section{Resultados}

En la figura 10 se muestran los resultados obtenidos de la medición de pulso cardiaco en la computadora.

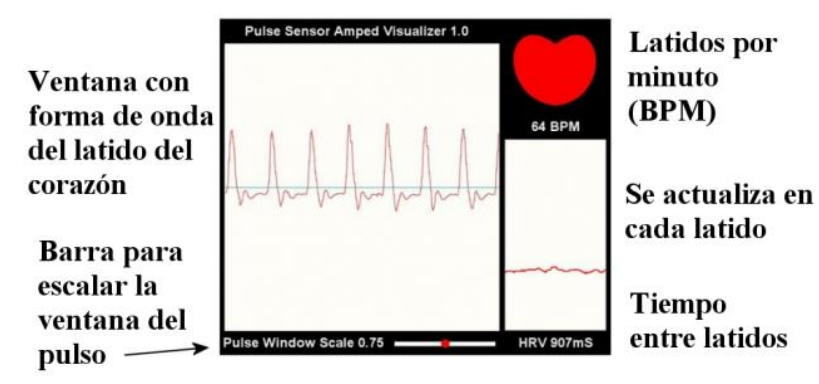

Figura 10 Grafica de pulso cardiaco de proccessing

Esta imagen muestra la gráfica de pulso cardiaco de la señal ya acondicionada, en pulsos por minuto (BPM), este monitoreo se genera por el programa llamado porccessing el cual es de dominio público y fue desarrollado por el M.I.T para simulaciones graficas biológicas y adaptado para mostrar gráficamente los datos. Con esta información el medico podrá tener un historial del paciente.

\section{Trabajo a futuro}

El microcontrolador Arduino tiene la capacidad de integrar más sensores, siendo estos, el de temperatura, presión arterial, etc. y pudiendo ser capaz de enviar esta información a distancia.
Adicionalmente se utilizara una investigación del Dr. Henri Poincaré que permite tener un diagnóstico de padecimientos cardiacos.

\section{Conclusiones}

Las comprobaciones prácticas funcionaron, $\mathrm{Se}$ logró la implementación del circuito que acondiciona el sensor para obtener una gráfica aceptable para futuros diagnósticos y a su vez su monitoreo en una computadora con el fin de dar a conocer estos resultados en forma remota o para llevar un historial de las mediciones, y ser analizados por un especialista médico y diagnostique posibles padecimientos.

\section{Referencias}

Boylestad, R \& Nashelsky, L. (2003). Electrónica: teoría de circuitos y dispositivos electrónicos. México: Pearson.

Corona, L., Abarca, G., Mares, J. (2014). sensores y Actuadores aplicaciones con Arduino. Mexico: Patria.

De Hoyos, F., Carreño, M., Serrano, E., Flores, N., \& Ríos, M. (2019). Development of a novel non-dispersive infrared multi sensor for measurement of gases in sediments. Sensors and Actuators B: Chemical, 288, 486-492.

Medran, R. . (2018). Medición del ritmo cardíaco mediante Fotopletismografía . Dpto. Teoría de la Señal y Comunicaciones Escuela Técnica Superior de Ingeniería Universidad de Sevilla: Universidad de Sevilla.

Mejía, H. \& Mejía, M... (2012). Oximetría de pulso. Revista de la Sociedad Boliviana de Pediatría, 5, pp 149-155.

Schlessinger, M. (2019). Infrared technology fundamentals. Routledge.

Ventura, V. (2015). Principio de funcionamiento del oxímetro para monitorización del pulso. Septiembre 11, 2019, de Polaridad.es Sitio web: https://polaridad.es/monitorizacion-sensorpulso-oximetro-frecuencia-cardiaca/ 
Yin, X., Wu, H., Dong, L., Ma, W., Zhang, L., Yin, W., \& Tittel, F. K. (2018). Ppb-level photoacoustic sensor system for saturation-free CO detection of SF6 decomposition by use of a $10 \mathrm{~W}$ fiber-amplified near-infrared diode laser. Sensors and Actuators B: Chemical, 282, 567573. 